\title{
ON THE HOMOLOGY OF ASSOCIATIVE ALGEBRAS
}

\author{
DAVID J. ANICK
}

\begin{abstract}
We present a new free resolution for $k$ as an $G$-module, where $G$ is an associative augmented algebra over a field $k$. The resolution reflects the combinatorial properties of $G$.
\end{abstract}

Introduction. Let $k$ be a field and let $G$ be an associative augmented $k$-algebra. For many purposes one wishes to have a projective resolution of $k$ as a $G$-module. The bar resolution is always easy to define, but it is often too large to use in practice. At the other extreme, minimal resolutions may exist, but they are often hard to write down in a way that is amenable to calculations. The main theorem of this paper presents a compromise resolution. Though rarely minimal, it is small enough to offer some bounds but explicit enough to facilitate calculations. As it relies heavily upon combinatorial constructions, it is best suited for analyzing otherwise tricky algebras given via generators and relations.

Since several results we get as consequences of the main theorem have been obtained before through other means, this paper may be viewed as generalizing and unifying several seemingly unrelated ideas. In particular, we are generalizing Priddy's results on Koszul algebras [12], extending homology computations by Govorov [9] and Backelin [3], and complementing Bergman's methods regarding the diamond lemma [6].

Three results may be of interest. The homology of the $\bmod p$ Steenrod algebra is given in terms of the homology of a new chain complex smaller than the $\Lambda$-algebra in Theorem 3.5. Formula (16) offers an efficient algorithm for the determination of Hilbert series, and Theorem 4.2 asserts the existence of new bounds on the torsion groups of commutative graded rings.

1. Definitions and the main theorem. Throughout this paper, $k$ denotes any field and $G$ is an associative $k$-algebra with unity. The field $k$ embeds in $G$ via $\eta: k \hookrightarrow G$ and we suppose that $G$ has an augmentation, i.e., a $k$-algebra map $\varepsilon: G \rightarrow k$ for which $\eta$ is a right inverse. $S$ denotes a set of generators for $G$ as a $k$-algebra and $k\langle S\rangle$ is the free associative $k$-algebra with unity on $S$. There is a canonical surjection $f: k\langle S\rangle \rightarrow G$ once $S$ is chosen, and the augmentation $\varepsilon$ is determined once we know $\varepsilon(x)$ for each $x \in S$. In particular, this means that $k\langle S\rangle$ may be augmented such that $f$ becomes a map of augmented algebras.

To $S$ we associate a function $e: S \rightarrow \mathbf{Z}_{+}$called a grading. In the absence of a more compelling choice we often take $e$ to be grading by length, i.e., $e(x)=1$ for

Received by the editors May 23, 1983 and, in revised form, February 22, 1984. This paper was the subject of an invited one-hour address in Boulder, Colorado, during the week-long AMS summer program on Combinatorics and Algebra, June 1983.

1980 Mathematics Subject Classification (1985 Revision). Primary 16A62; Secondary 13D03, $55 \mathrm{~S} 10$. 
all $x \in S$. $e$ extends uniquely to a map of monoids $e: B \rightarrow \mathbf{Z}_{+} \cup\{0\}$, where $B$ is the free monoid generated by $S$, and makes $k\langle S\rangle \approx \operatorname{Span}(B)$ into a graded algebra. We do not necessarily assume that $G$ inherits this gradation.

With the goal of selecting a vector space basis for $G$, let $<_{0}$ be a total ordering on the set $S$. We may define a total ordering on the monoid $B$ by setting $x<$ $y$ if $e(x)<e(y)$ and by resorting to the lexicographic ordering induced by $<_{0}$ when $e(x)=e(y)$. Because $<$ restricted to $S$ need not equal $<_{0}$, we maintain the distinction between them. We say that $\left(S, e,<_{0}\right)$ is locally well-ordered if and only if $e^{-1}(n) \cap S$ is well-ordered under $<_{0}$ for each $n$. An important special case occurs when $e^{-1}(n) \cap S$ is finite for each $n$, and then we describe $\left(S, e,<_{0}\right)$ as locally finite. It is a simple exercise to show that $(B,<)$ is well-ordered if and only if $\left(S, e,<_{0}\right)$ is locally well-ordered.

For $u, v \in B$, we say $v$ is a submonomial of $u$ and write $v \subseteq u$ if and only if $v=1$ or $u=x_{i_{1}} \cdots x_{i_{t}}$ and $v=x_{i_{m}} \cdots x_{i_{s}}$ for some $1 \leq m \leq s \leq t$ and $x_{i_{j}} \in S$. "Submonomial of" is a partial ordering on $B$ and we say that a subset $M \subseteq B$ is an order ideal of monomials (henceforth abbreviated "o.i.m.") if and only if $u \in M$ and $v \subseteq u$ imply $v \in M$. The following lemma generalizes Lemma 1.1 of [1]. The proof is obtained by extending the proof in [1] from ordinary to transfinite induction.

LEMMA 1.1. Let $\left(S, e,<_{0}\right)$ be locally well-ordered and $f: k\langle S\rangle \rightarrow G$ a surjection of $k$-algebras. The set $M=\{x \in B \mid f(x) \notin \operatorname{Span}(f(y) \mid y<x)\}$ is an o.i.m. and the elements $f(x)$ for $x \in M$ form a basis for $G$ as a $k$-module.

Given any o.i.m. $M$ in a free monoid $B$, define the set of obstructions for $M$ to be $V=V_{M}=\{v \in B \mid v \notin M$ but $u \varsubsetneqq v$ implies $u \in M\}$. Clearly $u \varsubsetneqq v$ and $u, v \in V$ cannot occur, i.e., $V$ is an antichain in $B$.

LEMMA 1.2. For $M$ an o.i.m. in a free monoid $B, x \in B-M$ if and only if $x$ contains as a submonomial some obstruction $v \in V_{M}$.

Proof. That $v \in V_{M}$ and $v \subseteq x$ imply $x \notin M$ is clear since $v \notin M$. Conversely, for $x \notin M$, let $v$ be a submonomial of $x$ of minimal length such that $v \notin M$. Proper submonomials of $v$ belong to $M$, hence $v \in V_{M}$.

Thus an o.i.m. in $B$ uniquely determines its obstruction antichain and any antichain $V$ uniquely determines an o.i.m. $M \subseteq B$ for which $V=V_{M}$. The correspondence between order ideals and antichains is actually valid on any partially ordered set having only finite descending chains. We may combine Lemmas 1.1 and 1.2 to describe a "straightening law" for $G$. Let $M$ be the o.i.m. of Lemma 1.1 and call a monomial $x$ admissible if and only if $x \in M$. For any $x \in B-M, f(x)$ may be expressed as a linear combination of $f$-images of smaller (in the sense of $<$ ) monomials and uniquely as a linear combination of $f$-images of admissible monomials. In particular, the obstructions may be so expressed. Conversely, suppose we know for each $v \in V_{M}$ how to write $f(v)=\sum_{x \in M ; x<v} c_{v x} f(x)$, where $c_{v x} \in k$ and for every $v$ almost all $c_{v x}$ are zero. Any $y \in B-M$ must equal $u v w$ for some $v \in V_{M}$ and $u, w \in B$, so we may write $f(y)$ as a linear combination of $f$-images of smaller monomials, $f(y)=\sum_{x \in M ; x<v} c_{v x} f(u x w)$, and apply this procedure recursively to each summand until we have $f(y)$ equaling some linear combination of $f(M)$. Since $(B,<)$ is well-ordered, this procedure must terminate after finitely many steps, and the resulting linear combination is unique because $f(M)$ is a basis. 
Our next objective is to show that the obstructions may be combined into a free $G$-resolution of $k$. First we select certain sets of monomials which will comprise the free $G$-basis. Let $V$ be any nontrivial (i.e., $1 \notin V$ ) antichain in the free monoid $B$ on a set $S$. For $n \geq 1, u=x_{i_{1}} \cdots x_{i_{t}} \in B$ is an $n$-prechain if and only if there exist integers $a_{j}$ and $b_{j}, 1 \leq j \leq n$, satisfying

(a) $1=a_{1}<a_{2} \leq b_{1}<a_{3} \leq b_{2}<\cdots<a_{n} \leq b_{n-1}<b_{n}=t$ and

(b) $x_{i_{a_{j}}} \cdots x_{i_{b_{j}}} \in V$ for $1 \leq j \leq n$.

Conceptually, an $n$-prechain is a sequence of $n$ not necessarily different elements of $V$, the end part of each monomial overlapping with the beginning part of the next. We also require that each monomial intersect only its immediate neighbors. The $n$-prechain $x_{i_{1}} \cdots x_{i_{t}}$ is an $n$-chain if and only if the integers $\left\{a_{j}, b_{j}\right\}$ may be chosen so as to satisfy

(c) $x_{i_{1}} \cdots x_{i_{s}}$ is not an $m$-prechain for any $s<b_{m}, 1 \leq m \leq n$.

For formal reasons it is convenient to define a 0 -chain to be any element of $S$, a $(-1)$-chain to be the minimal monomial $1 \in B$, and to let $b_{0}, b_{-1}$ be the integers 1 and 0 respectively. A 1 -chain is precisely an element of $V$ because $a_{1}=1$ and $b_{1}=t$ mean by (b) that $x_{i_{1}} \cdots x_{i_{t}} \in V$.

Some examples will help make clear this notion of $n$-chain. Suppose $V$ is the singleton $\left\{x^{3}\right\}, x \in S$. Then $x^{3}$ is a 1-chan $\left(a_{1}=1<b_{1}=3\right)$ and $x^{4}=x \sqrt{x x}$, viewed as two overlapping copies of $x^{3}$, is a 2-chain $\left(a_{1}=1<a_{2}=2 \leq b_{1}=3<\right.$ $\left.b_{2}=4\right) . x_{6}=x x x x x$ is a 3 -chain $(1<2 \leq 3<4 \leq 4<6)$ but $x^{5}=x x x x$ is not a 3-chain because it would violate $b_{1}<a_{3} \cdot x^{7}=x x x x x x$ is a 3-prechain but not a 3 -chain because, as we have already noted, the submonomial $x^{6}$ is a 3 -chain. Here $x^{7}=x x x_{x} x x$ is the only 4-chain, $x^{9}$ is the unique 5 -chain, and in general $x^{3 n}$ is a $(2 n-1)$-chain while $x^{3 n+1}$ is a $(2 n)$-chain.

As a second example suppose $V=\left\{x^{2} y x y, x y x y^{2}\right\}$. Then $x^{2} y x y^{2}=x x y x y y$ and xxy xyxyy are 2-chains but there are no $n$-chains for $n \geq 3$.

LEMMA 1.3. Let $B$ be the free monoid on $S$, and suppose $V \subseteq B$ is any nontrivial antichain. If $n \geq 1$ and $u=x_{i_{1}} \cdots x_{i_{t}}$ is an $n$-chain on $V$, then the integers $\left\{a_{j}, b_{j}\right\}$ fulfilling (a)-(c) above are uniquely determined. In particular, there is a unique $s=b_{n-1} \leq t$ such that $x_{i_{1}} \cdots x_{i_{s}}$ is an $(n-1)$-chain, and for this $s, x_{i_{s+1}} \cdots x_{i_{t}}$ does not contain as a submonomial any element of $V$.

PROOF. Suppose $\left\{a_{j}, b_{j}\right\}$ and $\left\{a_{j}^{\prime}, b_{j}^{\prime}\right\}$ could both serve in (a) and (b) above for some $u=x_{i_{1}} \cdots x_{i_{t}}$. Then each $b_{m}=b_{m}^{\prime}$, or else the larger one would violate (c). That $a_{m}=a_{m}^{\prime}$ follows because $x_{i_{a_{m}}} \cdots x_{i_{b_{m}}}$ and $x_{i_{a_{m}^{\prime}}} \cdots x_{i_{b_{m}}}$ must both lie in the antichain $V$. Properties (a)-(c) are clearly inherited by $x_{i_{1}} \cdots x_{i_{b_{m}}}$ for $1 \leq m \leq n$.

For $U$ a subset of $B$, let $U k$ denote the $k$-submodule of $k\langle S\rangle$ spanned by $U$. For $G$ a $k$-algebra and $M$ as in Lemma 1.1, $U k \otimes G$ (" $\otimes$ " means " $\otimes_{k}$ ") has as a basis $\hat{U}=\{u \otimes f(x) \mid u \in U, x \in M\}$. When no confusion can result we denote the element $u \otimes f(x)$ of $\hat{U}$ by $u \otimes x$.

Define a partial order on $\hat{U}$ by writing $u \otimes x<u^{\prime} \otimes x^{\prime}$ if and only if $u x<u^{\prime} x^{\prime}$ in $B$. We will be interested in $\hat{U}$ when $U$ is the set of $n$-chains on the antichain $V$. In that case $u x=u^{\prime} x^{\prime}$ is impossible by property (c) unless $u=u^{\prime}$ and then $x=x^{\prime}$, so $<$ becomes a total order on $U$. When $w=\sum_{j=1}^{g} c_{j}\left(u_{j} \otimes v_{j}\right) \in U k \otimes G$ with $c_{j} \in k-(0), u_{j} \in U, v_{j} \in M$, we say that $u_{1} \otimes v_{1}$ is the high term of $w$ if and only 
if $q=1$ or $u_{1} \otimes v_{1}>u_{j} \otimes v_{j}$ for all $j \neq 1$. Such a situation will also be denoted "HT $(w)=u_{1} v_{1}$."

We now factor the augmentation into the picture and prove the main theorem.

THEOREM 1.4. Let $G$ be an associative augmented $k$-algebra, generated as a $k$-algebra by the locally well-ordered set $\left(S, e,<_{0}\right)$. Let $M$ be the o.i.m. of Lemma 1.1 , let $V$ be the set of obstructions for $M$, and let $V^{(n)}$ be the set of $n$-chains on $V$. Then there is a free $G$-resolution of $k$,

$$
0 \leftarrow k \stackrel{\varepsilon}{\leftarrow} G \stackrel{\delta_{0}}{\leftarrow} S k \otimes G \stackrel{\delta_{1}}{\leftarrow} V k \otimes G \stackrel{\delta_{2}}{\longleftarrow} V^{(2)} k \otimes G \stackrel{\delta_{3}}{\longleftarrow} V^{(3)} k \otimes G \leftarrow \cdots
$$

in which

$$
\delta_{0}(x \otimes 1)=x-\eta \varepsilon(x)
$$

for $x \in S$ and for $n \geq 1$,

$$
\delta_{n}\left(x_{i_{1}} \cdots x_{i_{b_{n}}} \otimes 1\right)=x_{i_{1}} \cdots x_{i_{b_{n-1}}} \otimes x_{i_{b_{n-1}+1}} \cdots x_{i_{b_{n}}}+\omega,
$$

where $H T(\omega)<x_{i_{1}} \cdots x_{i_{b_{n}}}$ if $\omega \neq 0$.

Proof. We must define $\delta_{n}$ and show that (1) is exact. Exactness at $G$ follows because $\varepsilon \delta_{0}=0$ and $\operatorname{ker}(\varepsilon)$ is spanned by $\{y-\eta \varepsilon(y) \mid y \in G\}$. If $y=f\left(x_{i_{1}} \cdots x_{i_{t}}\right)$, $x_{i_{1}} \cdots x_{i_{t}} \in M$, set

$$
\begin{aligned}
i_{0}(y-\eta \varepsilon(y))=x_{i_{1}} \otimes x_{i_{2}} \cdots x_{i_{t}}+\varepsilon\left(x_{i_{1}}\right)\left(x_{i_{2}} \otimes x_{i_{3}} \cdots x_{i_{t}}\right) & \\
& +\cdots+\varepsilon\left(x_{i_{1}} \cdots x_{i_{t-1}}\right)\left(x_{i_{t}} \otimes 1\right) .
\end{aligned}
$$

Then $i_{0}$ extends to a $k$-module map $i_{0}: \operatorname{ker}(\varepsilon) \rightarrow S k \otimes G$ with $\delta_{0} i_{0}=\operatorname{id}_{\operatorname{ker}(\varepsilon)}$. This means that $\operatorname{im} \delta_{0}=\operatorname{ker}(\varepsilon)$.

The sequence (1) may be rewritten, including $\eta$ and $i_{0}$, as

$$
0 \leftarrow k \underset{\eta}{\stackrel{\varepsilon=\delta_{-1}}{\leftrightarrows}} V^{(-1)} k \otimes G \underset{i_{0}}{\stackrel{\delta_{0}}{\leftrightarrows}} V^{(0)} k \otimes G \stackrel{\delta_{1}}{\longleftarrow} V^{(1)} k \otimes G \stackrel{\delta_{2}}{\leftarrow} V^{(2)} k \otimes G \leftarrow \cdots
$$

Suppose inductively that for some $n \geq 1,\left\{\delta_{j}\right\}$ have been defined satisfying (2) or (3) for $0 \leq j<n$ and that the sequence (4) has been proved exact to the left of $V^{(n-1)} k \otimes G$. Suppose further that there are $k$-module splittings $i_{j}: \operatorname{ker}\left(\delta_{j-1}\right) \rightarrow$ $V^{(j)} k \otimes G$ for $0 \leq j<n$ satisfying

$$
\delta_{j} i_{j}=\mathrm{id}_{\mathrm{ker}\left(\delta_{j-1}\right)}
$$

and

$$
H T\left(i_{j}(\omega)\right)=H T(\omega)
$$

These properties hold for $j=0$, so it suffices to verify them for $j=n$ when they hold for $j<n$.

First we define $\delta_{n}$. As $\delta_{n}$ is to be a $G$-module map, we need only specify $\delta_{n}(u \otimes 1)$ for $u \in V^{(n)}$. By Lemma 1.3 we may uniquely write $u=r s$, where $r \in V^{(n-1)}$ and $s \in M$. Consider $\delta_{n-1}(r \otimes s)=\delta_{n-1}(r) \cdot s$. Writing $u=x_{i_{1}} \cdots x_{i_{b_{n}}}$, we have by the inductive assumption

$$
\begin{aligned}
\delta_{n-1}(r) \cdot s & =\left(x_{i_{1}} \cdots x_{i_{b_{n-2}}} \otimes x_{i_{b_{n-2}+1}} \cdots x_{i_{b_{n-1}}}\right)\left(x_{i_{b_{n-1}+1}} \cdots x_{i_{b_{n}}}\right)+\omega \\
& =x_{i_{1}} \cdots x_{i_{b_{n-2}}} \otimes f\left(x_{i_{b_{n-2}+1}} \cdots x_{i_{b_{n}}}\right)+\omega
\end{aligned}
$$


where $H T(\omega)<u$. Since $x_{i_{b_{n-2}+1}} \cdots x_{i_{b_{n}}}$ has the obstruction $x_{i_{a_{n}}} \cdots x_{i_{b_{n}}}$ as a submonomial, however, the entire right-hand side of (7) has high term less than $u$. Inductively $\sigma=i_{n-1} \delta_{n-1}(r \otimes s)$ has been defined, implying that $\delta_{n-1}(\sigma)=$ $\delta_{n-1}(r \otimes s)$ while by $(6) H T(\sigma)<u$. Define $\delta_{n}(u)$ to be

$$
\delta_{n}(u \otimes 1)=r \otimes s-\sigma .
$$

Then $\delta_{n}$ fulfills formula $(3)$ and $\delta_{n-1} \delta_{n}(u \otimes 1)=0$.

The splitting $i_{n}=\operatorname{ker}\left(\delta_{n-1}\right) \rightarrow V^{(n)} k \otimes G$ is defined recursively, so it is here that we make use of the fact that $(B,<)$ and consequently $\left(\hat{V}^{(n-1)},<\right)$ are well-ordered. Suppose

$$
\omega=\sum_{j=1}^{q} c_{j}\left(u_{j} \otimes v_{j}\right) \in \operatorname{ker}\left(\delta_{n-1}\right),
$$

that $u_{1} \otimes v_{1}$ is the high term of $\omega$, and that $i_{n}\left(\omega^{\prime}\right)$ has been defined and satisfies (5) and (6) for $\omega^{\prime} \in \operatorname{ker}\left(\delta_{n-1}\right)$ with $H T\left(\omega^{\prime}\right)<u_{1} v_{1}$. Because $\omega \in \operatorname{ker}\left(\delta_{n-1}\right)$ we must by $(3)$ have $H T\left(\delta_{n-1}\left(u_{1} \otimes v_{1}\right)\right)<u_{1} v_{1}$. Writing $u_{1}=x_{i_{1}} \cdots x_{i_{b_{n-1}}}$ and $v_{1}=x_{i_{b_{n-1}+1}} \cdots x_{i_{s}}$

$$
H T\left(x_{i_{1}} \cdots x_{i_{b_{n-2}}} \otimes f\left(x_{i_{b_{n-2}+1}} \cdots x_{i_{b_{n-1}}} x_{i_{b_{n-1}+1}} \cdots x_{i_{s}}\right)\right)<u_{1} v_{1}
$$

which can only happen if $x_{i_{b_{n-2}+1}} \cdots x_{i_{s}}$ contains some obstruction. Letting $x_{i_{a_{n}}} \ldots$ $x_{i_{b_{n}}}$ be the obstruction contained in $x_{i_{b_{n-2}+1}} \cdots x_{i_{s}}$ which starts furthest to the left, we must have $b_{n-2}<a_{n} \leq b_{n-1}<b_{n}$, which makes $x_{i_{1}} \cdots x_{i_{b_{n}}}$ an $n$-chain. Furthermore, $y=x_{i_{n}+1} \cdots x_{i s}$ is a submonomial of $v_{1} \in M$, so $y \in M$. This shows that

$$
\tau=x_{i_{1}} \cdots x_{i_{n}} \otimes y \in V^{(n)} k \otimes G .
$$

By (3), $u_{1} \otimes v_{1}$ is the high term of $\delta_{n}(\tau)$, so $\omega^{\prime}=\omega-c_{1} \delta_{n}(\tau) \in \operatorname{ker}\left(\delta_{n-1}\right)$ while $H T\left(\omega^{\prime}\right)<u_{1} v_{1}$. Define $i_{n}(\omega)$ by

$$
i_{n}(\omega)=c_{1} \tau+i_{n}\left(\omega^{\prime}\right)
$$

Then (6) is fulfilled and $\delta_{n} i_{n}(\omega)=c_{1} \delta_{n}(\tau)+\omega^{\prime}=\omega$. The $k$-linearity of $i_{n}$ is automatic. This completes the recursive step in the definition of $i_{n}$. In particular, $\delta_{n}$ is onto $\operatorname{ker}\left(\delta_{n-1}\right)$, i.e., the sequence (4) is exact at $V^{(n-1)} k \otimes G$.

We wish to specialize Theorem 1.4 to the case of connected graded $k$-algebras. An augmented $k$-algebra $G$ is a connected graded $k$-algebra if and only if there are $k$-submodules $\left\{G_{n}\right\}_{n \geq 0}$ such that $G \approx \bigoplus_{n=0}^{\infty} G_{n}$, where $G_{0}=\operatorname{im}(\eta) \approx k$, $\operatorname{ker}(\varepsilon)=\bigoplus_{n=1}^{\infty} G_{n}$, and $\left(G_{m}\right) \cdot\left(G_{n}\right) \subseteq G_{m+n}$. An element $x \in G$ is homogeneous if and only if $x \in G_{n}$ for some $n$, in which case we write $|x|=n$. We have already alluded to the fact that $H=k\langle S\rangle$ is graded, with $H_{n}=\operatorname{Span}\{x \in B \mid e(x)=n\}$. Since any graded algebra has generating sets consisting of homogeneous elements, we will always assume when $G$ is graded that $|f(x)|=e(x)$ for $x \in S$. Then the surjection $f: k\langle S\rangle \rightarrow G$ is a map of connected graded algebras, i.e., $|f(x)|=n$ whenever $|x|=n$.

Since each $x \in B$ is homogeneous in $H=k\langle S\rangle$ and the $\left\{G_{n}\right\}$ are linearly independent, $f(x) \in \operatorname{Span}\{f(y) \mid y<x, y \in B\}$ if and only if $f(x) \in \operatorname{Span}\{f(y) \mid e(y)=$ $e(x)$ and $y<x, y \in B\}$. In other words, not only does the o.i.m. $M$ of Lemma 1.1 consist of homogeneous elements in the graded case, but also the straightening 
law for any obstruction is homogeneous. If $v \in V_{M}$ and $f(v)=\sum_{x \in M ; x<v} c_{v x} f(x)$, then $c_{v x}=0$ for $e(x) \neq e(v)$. The $k$-modules $V^{(n)} k$ and consequently $V^{(n)} k \otimes G$ inherit gradings from that of $H$. These observations allow us to deduce the graded version of Theorem 1.4, which we state without further proof.

THEOREM 1.5. If $G$ is a connected graded $k$-algebra, then each homomorphism in the exact sequence (1) is a homomorphism of graded $G$-modules. In particular, the right-hand side of $(3)$ is homogeneous, with $|\omega|=\left|x_{i_{1}}\right|+\cdots+\left|x_{i_{b_{n}}}\right|$.

2. The diamond lemma revisited. We take up here the practical matter of determining the set of obstructions for an algebra given in terms of generators and relations. The differential $\delta_{2}$ in (1) is shown to reflect the process of resolving overlap ambiguities discussed in [6]. We also observe that the obstruction set is recursive for a locally finite graded algebra.

Many authors have studied algebras from the point of view taken here, which variously emphasizes normal form for elements, straightening laws, or unambiguous reduction. We adopt [6] as our primary reference. Given an o.i.m. $M \subseteq B$ and its obstruction antichain $V$, with $B$ the free monoid on the locally well-ordered graded set $\left(S, e,<_{0}\right)$, we can identify $M k$ with a unique quotient $G$ of $k\langle S\rangle$ if we have a function $\tau: V \rightarrow M k$ such that $\tau(x) \in \operatorname{Span}\{y \in M \mid y<x\}$, and such that $\tau$ has no overlap ambiguities. The latter condition requires that repeated application of the maps given by $u v w \mapsto u \tau(v) w$ for $v \in V$ and $y \mapsto y$ for $y \in M$ stabilizes pointwise at something we call $h_{\tau}$, and that $h_{\tau}(x)$ is independent of the order or choice of maps. Recursively, this is equivalent to requiring whenever $x_{i_{1}} \cdots x_{i_{b_{2}}}$ is a 2-prechain, that

$$
h_{\tau}\left(x_{i_{1}} \cdots x_{i_{a_{2}-1}} \tau\left(x_{i_{a_{2}}} \cdots x_{i_{b_{2}}}\right)\right)=h_{\tau}\left(\tau\left(x_{i_{1}} \cdots x_{i_{b_{1}}}\right) x_{i_{b_{1}+1}} \cdots x_{i_{b_{2}}}\right) .
$$

The use of an antichain for $V$ eliminates the possibility of inclusion ambiguities, which would otherwise also occur (see [6]).

In our context, we can immediately relax the requirement for $\tau$ from being ambiguity-free on 2-prechains to 2-chains. For suppose the reduction scheme $n_{\tau}$ defined by $\tau$ is unambiguous on 2-chains, and let $w=x_{i_{1}} \cdots x_{i_{b_{2}}}$ be the smallest 2-prechain for which

$$
u_{1}=\tau\left(x_{i_{1}} \cdots x_{i_{b_{1}}}\right) x_{i_{b_{1}+1}} \cdots x_{i_{b_{2}}} \text { and } \quad u_{3}=x_{i_{1}} \cdots x_{i_{a_{2}-1}} \tau\left(x_{i_{a_{2}}} \cdots x_{i_{b_{2}}}\right)
$$

are straightened differently. Since $w$ is not a 2-chain, there is a 2-chain $w^{\prime}=$ $x_{i_{1}} \cdots x_{i_{s}}$ with $s<b_{2}$ and we let $r$ be the index for which $x_{i_{r}} \cdots x_{i_{s}} \in V . w^{\prime \prime}=$ $x_{i_{r}} \cdots x_{i_{b_{2}}}$ is also a 2-prechain and $w^{\prime \prime}<w$, so

$$
h_{\tau}\left(\tau\left(x_{i_{r}} \cdots x_{i_{s}}\right) x_{i_{s+1}} \cdots x_{i_{b_{2}}}\right)=h_{\tau}\left(x_{i_{r}} \cdots x_{i_{a_{2}-1}} \tau\left(x_{i_{a_{2}}} \cdots x_{i_{b_{2}}}\right)\right) \text {. }
$$

Note that $u_{2}=x_{i_{1}} \cdots x_{i_{r-1}} \tau\left(x_{i_{r}} \cdots x_{i_{s}}\right) x_{i_{s+1}} \cdots x_{i_{b_{2}}}$ and $u_{3}$ are both spanned by monomials lower than $w$, so $h_{\tau}$ is uniquely defined on each of them. In particular,

$$
\begin{aligned}
h_{\tau}\left(u_{2}\right) & =h_{\tau}\left(x_{i_{1}} \cdots x_{i_{r-1}} h_{\tau}\left(\tau\left(x_{i_{r}} \cdots x_{i_{s}}\right) x_{i_{s_{+}+1}} \cdots x_{i_{b_{2}}}\right)\right) \\
& =h_{\tau}\left(x_{i_{1}} \cdots x_{i_{r-1}} h_{\tau}\left(x_{i_{r}} \cdots x_{i_{a_{2}-1}} \tau\left(x_{i_{a_{2}}} \cdots x_{i_{b_{2}}}\right)\right)\right) \\
& =h_{\tau}\left(u_{3}\right),
\end{aligned}
$$

and likewise $h_{\tau}\left(u_{1}\right)=h_{\tau}\left(u_{2}\right)$. Consequently $h_{\tau}\left(u_{1}\right)=h_{\tau}\left(u_{3}\right)$, contradicting our choice of $w$. 
When $S$ is locally finite, we review next an algorithm for finding or approximating the obstruction set $V$, given that $G$ is the quotient of $k\langle S\rangle$ by the finite or countable relation set $\left\{\rho_{i}\right\}_{i \geq 1}$. Write each relation in the form $\rho_{i}=r_{i}-\sum_{j=1}^{t_{i}} c_{i j} r_{i j}$, where $r_{i}, r_{i j} \in B, c_{i j} \in \bar{k}$, and $r_{i}>r_{i j}$ for all $i$ and $j$; it simplifies matters to assume that the $\left\{\rho_{i}\right\}_{i \geq 1}$ are arranged such that $r_{1} \leq r_{2} \leq r_{3} \leq \cdots$. We will construct a sequence $\left\{V_{n}, \tau_{n}\right\}$ of antichains $V_{n} \subseteq B$ and functions $\tau_{n}: V_{n} \rightarrow M_{n} k$, where $V_{n}=V_{\left(M_{n}\right)}$ as in Lemma 1.2, and $\tau_{n}(v) \in \operatorname{Span}\left\{x \in M_{n} \mid x<v\right\}$ for each $v \in V_{n}$. From a set theoretic standpoint, we think of $\left\{\tau_{n}\right\}$ as functions from a subset of $B$ to a subset of $B k$, hence as subsets of $B \times B k$.

Begin the algorithm by setting $U_{n}=\left\{r_{i} \mid e\left(r_{i}\right)=n\right\}$ and $\tilde{\tau}\left(r_{i}\right)=\sum_{j=1}^{t_{i}} c_{i j} r_{i j} \in$ $B k$. Let $\left(V_{0}, \tau_{0}\right)$ be empty.

Suppose $\left(V_{n}, \tau_{n}\right)$ has been constructed and has the property that $\tau_{n}$ defines a unique reduction scheme $h_{\tau_{n}}:\{x \in B \mid e(x) \leq n\} \rightarrow B k$ and furthermore that $f\left(x-h_{\tau_{n}}(x)\right)=0$ on this set, where $f: k\langle S\rangle=B k \rightarrow G$ is the canonical surjection. (When $\tau_{n}$ is empty, $h_{\tau_{n}}$ is defined by $h_{\tau_{n}}(x)=x$ for all $x \in B$.) To define $\left(V_{n+1}, \tau_{n+1}\right)$, start with $\left(V^{\prime}, \tau^{\prime}\right)=\left(V_{n}, \tau_{n}\right) \cup\left(U_{n+1},\left.\tilde{\tau}\right|_{U_{n+1}}\right)$. Let $w$ be the least monomial (in the sense of $<$ ) if any for which $h_{\tau^{\prime}}(w)$ has two possible values $y_{1}$ and $y_{2}$. To resolve the ambiguity, treat $y_{1}-y_{2}$ as a new relation in adjoining its high term $y$ to $V^{\prime}$, and define $\tau^{\prime}(y)$ in accordance with the requirement that $h_{\tau^{\prime}}\left(y_{1}-y_{2}\right)$ become zero. Repeat this step until $h_{\tau^{\prime}}$ has no ambiguities in degrees $\leq n+1$. Only finitely many steps will be needed, as the local finiteness of $S$ guarantees that only finitely many monomials are involved at this stage. Take $V_{n+1}$ to be the set of minimal (in the sense of $\subseteq$ ) monomials in the final $V^{\prime}$, and $\tau_{n+1}$ to be the reduction scheme $h_{\tau^{\prime}}$ obtained from the final $\tau^{\prime}$. Because of the descending chain condition on $(B,<)$, the membership of the $\left\{V_{n}\right\}$ and the functions $\left\{\tau_{n}\right\}$ must stabilize pointwise, i.e.,

$$
(V, \tau)=\bigcap_{m \geq 1} \bigcup_{n \geq m}\left(V_{n}^{r}, \tau_{n}\right)
$$

is the set of obstructions together with a straightening rule for $G$. A word of warning: when $x \in V_{n}$, there is no assurance that $x$ will ultimately appear in $V$, since it may be undercut by having some proper submonomial fall in $V_{m}$ for $m>n$. The most we can say in general is that the set $B-M$, consisting of those monomials which contain some obstruction as a submonomial, is recursively enumerable.

An algebra $G$ has solvable word problem relative to the generating set $S$ if and only if, when $f: k\langle S\rangle \rightarrow G$ is the canonical map and $x, y$ are any two elements of $k\langle S\rangle$, there is an algorithm for deciding whether or not $f(x)=f(y)$. Assuming that $S$ is at most countable and has been given a locally finite grading, the associative $k$-algebra $G$ clearly has solvable word problem relative to $S$ if the set of obstructions is recursive, and the converse is true over finite fields $k$. The converse is also true for a group ring $k(P)$ if $S$ consists of a set of generators for $P$ and their inverses, since $k(P)$ has solvable word problem if and only if $P$ has solvable word problem in the group theory sense. Whether or not a solvable word problem implies a recursive obstruction set for arbitrary algebras over infinite fields remains open. For graded algebras, however, we have a complete answer.

THEOREM 2.1. Let $\left(S, e,<_{0}\right)$ be any locally finite ordered graded set, and suppose $\rho=\left\{\rho_{i}\right\}_{i \geq 1}$ is a finite or countable set of homogeneous elements in $k\langle S\rangle$. 
Suppose further that $\left|\rho_{1}\right| \leq\left|\rho_{2}\right| \leq \cdots$ and that $G$ is the quotient of $k\langle S\rangle$ by the twosided ideal $\rho$ generates. Then the obstruction set for $G$ is recursive. In particular, $G$ has solvable word problem relative to $\left(S, e,<_{0}\right)$.

Proof. When $G$ is graded, the $n$th stage in the above procedure generates and resolves ambiguities in degree $n$ only, and this does not affect $V_{n}$ or $\tau_{n}$ in degrees $<n$. If $x \in B$ has $e(x)=m$, then $x \in V$ if and only if $x \in V_{m}$. Thus we can decide in finitely many steps whether or not $x \in V$.

We wish to observe that the process of resolving overlap ambiguities, which obtains from a 2-chain either a new obstruction or (if there was no ambiguity) zero, is reflected in the map $\delta_{2}$ of (1) from 2-chains to 1-chains. Although this is true in general, it is clearest and most useful in the connected graded case, so we restrict our attention to that situation. In the process we shall give explicit formulas for the algorithm of Theorem 2.1 .

By omitting the term " $k$ " and tensoring the sequence (1) over $G$ with $k$ we obtain the chain complex

$$
0 \leftarrow k \stackrel{0}{\leftarrow} S k \stackrel{\bar{\delta}_{1}}{\leftarrow} V k \stackrel{\bar{\delta}_{2}}{\leftarrow} V^{(2)} k \stackrel{\bar{\delta}_{3}}{\longleftarrow} V^{(3)} k \leftarrow \cdots .
$$

We seek to calculate the map $\bar{\delta}_{2}$ explicitly. To do this, we first need to define several simple homomorphisms among the graded vector spaces $B k, M k$, and $V k$.

Define $\mu: B k \otimes B k \rightarrow B k$ to be $\mu(u \otimes v)=u v$. Let $\bar{B}=B-\{1\}$ and note that the splitting $i_{0}: \operatorname{ker}(\varepsilon) \rightarrow S k \otimes M k \hookrightarrow S k \otimes B k$ which occurs in the proof of Theorem 1.4 extends easily to $i_{0}: \bar{B} k \stackrel{\approx}{\rightarrow} S k \otimes B k$, where $i_{0}\left(x_{i_{1}} x_{i_{2}} \cdots x_{i_{t}}\right)=x_{i_{1}} \otimes x_{i_{2}} \cdots x_{i_{t}}$. Take $f: B k \rightarrow M k$ to be the straightening map $B k \stackrel{\approx}{\rightarrow} k\langle S\rangle \rightarrow G \stackrel{\approx}{\rightarrow} M k$, so $\left.f\right|_{M k}=\operatorname{id}_{(M k)}$. Define $h_{0}, h_{1}, h: B k \rightarrow V k \otimes B k$ by

$$
h_{0}(w)= \begin{cases}w \otimes 1 & \text { if } w \in V \\ 0 & \text { otherwise }\end{cases}
$$

and

$$
h_{1}(w)= \begin{cases}v \otimes u & \text { if } w=v u \text { with } v \in V \text { and } u \in \bar{B} \\ 0 & \text { otherwise, }\end{cases}
$$

and $h(w)=h_{0}(w)+h_{1}(w)$ for $w \in B$. Lastly, set $f_{L}=\mu \circ\left(f \underset{\tilde{f}_{0}}{\otimes}\right) \circ h_{1}, \tilde{f}_{L}=$ $\mu \circ(f \otimes 1) \circ h, f_{R}=\mu \circ(1 \otimes f) \circ i_{0}, f_{0}=f_{R} \circ f_{L}$, and $\tilde{f}_{0}=f_{R} \circ \tilde{f}_{L}$.

We seek $\delta_{2}$, we know $\delta_{0}$, and we note that

$$
\begin{aligned}
\delta_{1}(v \otimes u) & =\left(\delta_{1}(v \otimes 1)\right) u=\left(i_{0}(v-f(v))\right) u \\
& =(1 \otimes f) \circ i_{0}(v u-f(v) u) .
\end{aligned}
$$

In view of the proof of Theorem 1.4, we need next a formula for the splitting $i_{1}: \operatorname{ker}\left(\delta_{0}\right) \rightarrow V k \otimes G \approx V k \otimes M k$.

LEMMA 2.2. Define $j: S k \otimes M k \rightarrow V k \otimes M k$ by $j(x \otimes y)=\sum_{n \geq 0} h \circ\left(\tilde{f}_{0}\right)^{n}(x y)$. Then $\left.j\right|_{\operatorname{ker}\left(\delta_{0}\right)}$ equals the splitting $i_{1}: \operatorname{ker}\left(\delta_{0}\right) \rightarrow V k \otimes M k$ described in the proof of Theorem 1.4.

PROOF. First, we note that $\tilde{f}_{0}(u)$ for $u \in B$ is spanned by monomials strictly lower than $u$, so $\tilde{f}_{0}$ is pointwise nilpotent and the infinite sum converges. Now let $w \in \operatorname{ker}\left(\delta_{0}\right)$ and suppose we have shown that $j\left(w^{\prime}\right)=i_{1}\left(w^{\prime}\right)$ for $w^{\prime} \in \operatorname{ker}\left(\delta_{0}\right)$ when 
$H T\left(w^{\prime}\right)<H T(w)$. Let $x \otimes y$ be the high term of $w$, occurring with coefficient $c \in k-(0)$, and let $w_{0}=w-c(x \otimes y)$. We know that $h(x y) \neq 0$ so write $h(x y)=v \otimes u$. Using the inductive assumption and definition of $i_{1}$,

$$
\begin{aligned}
i_{1}\left(c^{-1} w\right) & =v \otimes u+i_{1}\left(c^{-1} w-\delta_{1}(v \otimes u)\right)=v \otimes u+j\left(c^{-1} w-\delta_{1}(v \otimes u)\right) \\
& =v \otimes u+j\left(c^{-1} w_{0}+x \otimes y-x \otimes y+(1 \otimes f) i_{0} \mu(f \otimes 1) h(x y)\right) \\
& =v \otimes u+j\left(c^{-1} w_{0}+i_{0} \tilde{f}_{0}(x y)\right) \\
& =h(x y)+j\left(c^{-1} w_{0}\right)+\sum_{n \geq 0} h\left(\tilde{f}_{0}\right)^{n} \tilde{f}_{0}(x y) \\
& =j\left(c^{-1} w_{0}\right)+\sum_{n \geq 0} h\left(\tilde{f}_{0}\right)^{n}(x y)=j\left(c^{-1} w\right),
\end{aligned}
$$

as desired.

LEMMA 2.3. For $y \in V^{(2)}$,

$$
\delta_{2}(y \otimes 1)=\sum_{n \geq 0} h \circ\left(f_{0}\right)^{n}\left(\mathrm{id}-f_{R}\right)(y) .
$$

ProOF. First observe that $h \tilde{f}_{0}(v)=0$ when $v \in V$. This follows because

$$
h \tilde{f}_{0}(v)=h f_{R} \mu(f \otimes 1)(v \otimes 1)=h f_{R} f(v)=h f(v) \in h(M k)=0 .
$$

So $h \tilde{f}_{0}=h f_{0}$, and

$$
h\left(\tilde{f}_{0}\right)^{n}=h\left(\tilde{f}_{0} \mu h\right)^{n}=\left(h \tilde{f}_{0} \mu\right)^{n} h=\left(h f_{0} \mu\right)^{n} h=h\left(f_{0} \mu h\right)^{n}=h\left(f_{0}\right)^{n},
$$

and then

$$
j=\sum_{n \geq 0} h\left(\tilde{f}_{0}\right)^{n} \mu=\sum_{n \geq 0} h\left(f_{0}\right)^{n} \mu .
$$

Using this and the definition of $\delta_{2}$,

$$
\begin{aligned}
\delta_{2}(y \otimes 1) & =h(y)-i_{1} \delta_{1}(h(y)) \\
& =h(y)-\sum_{n \geq 0} h\left(f_{0}\right)^{n} f_{R}(y)+\sum_{n \geq 0} h\left(f_{0}\right)^{n}\left(\tilde{f}_{0}\right)(y) \\
& =-\sum_{n \geq 0} h\left(f_{0}\right)^{n} f_{R}(y)+h(y)+\sum_{n \geq 0} h\left(f_{0}\right)^{n+1}(y) \\
& =\sum_{n \geq 0} h\left(f_{0}\right)^{n}\left(\mathrm{id}-f_{R}\right)(y),
\end{aligned}
$$

as claimed.

From Lemma 2.3 we deduce at once that with $\bar{S}_{2}$ as in (8),

$$
\bar{\delta}_{2}(y)=\sum_{n \geq 0} h_{0}\left(f_{0}\right)^{n}\left(\mathrm{id}-f_{R}\right)(y)
$$

for $y \in V^{(2)}$, an extremely useful closed form. Let us see how this relates to the algorithm of Theorem 2.1.

First we wish to simplify the infinite sum in (9). Let $\gamma: B k \rightarrow B k$ be given by $\gamma(w)=w-\mu h_{1}(w)$. Set $\zeta=\gamma+f_{L}$ and $\theta=f_{R} \circ \zeta$. In words, the map $\zeta$ instructs us to leave any monomial alone unless it properly contains an obstruction in the 
leftmost position, in which case we straighten that obstruction. The composition $\theta^{n}$ tells us to alternate repeatedly between that operation and the operation which straightens any obstructions except those in the leftmost slot. Monomials not left fixed by $\theta$ are sent to linear combinations of strictly smaller monomials, which means that the sequence $\theta, \theta^{2}, \theta^{3}, \cdots$ stabilizes pointwise. Let $\xi=\lim _{n \rightarrow \infty} \theta^{n}$.

LEMMA 2.4. If $y \in \operatorname{im}\left(f_{R}\right)+\operatorname{im}\left(\mu h_{1}\right) \subseteq B k$, then

$$
h_{0} \theta^{n}(y)=\sum_{m=0}^{n} h_{0}\left(f_{0}\right)^{m}(y) \quad \text { and } \quad h_{0} \xi(y)=\sum_{m=0}^{\infty} h_{0}\left(f_{0}\right)^{m}(y) .
$$

PROOF. Note that $f_{0} \gamma=0$, that $h_{0} \gamma=h_{0}$, and for $y \in \operatorname{im}\left(f_{R}\right)+\operatorname{im}\left(\mu h_{1}\right)$ that $\theta(y)=\left(f_{0}+\gamma\right)(y)$. On the subspace $\operatorname{im}\left(f_{R}\right)+\operatorname{im}\left(\mu h_{1}\right)$, which is fixed by $\theta, f_{0}$, and $\gamma$, we have

$$
\theta^{n}=\left(f_{0}+\gamma\right)^{n}=f_{0}^{n}+\gamma f_{0}^{n-1}+\gamma^{2} f_{0}^{n-2}+\cdots+\gamma^{n}
$$

from which

$$
h_{0} \theta^{n}=h_{0}\left(f_{0}\right)^{n}+h_{0}\left(f_{0}\right)^{n-1}+\cdots+h_{0} .
$$

Letting $n \rightarrow \infty$ yields the latter formula.

When $y$ is a 2-chain, we automatically have $y \in \operatorname{im}\left(\mu h_{1}\right)$ and $f_{R}(y) \in \operatorname{im}\left(f_{R}\right)$, so (9) can be rewritten as

$$
\bar{\delta}_{2}(y)=h_{0}\left(\xi(y)-\xi f_{R}(y)\right) .
$$

Suppose for some $n \geq 1$ that we have found the set $V_{n}$ of obstructions of degree $\leq n$, along with their straightening rules $\tau_{n}: V_{n} \rightarrow M_{n} k$, where $M_{n}=\{x \in B \mid x$ has no $v \in V_{n}$ as a submonomial $\}$. This information suffices to compute $z=$ $\xi(y)-\xi f_{R}(y)$ for any 2-chain $y$ having $e(y)=n+1$. The connection with the algorithm of Theorem 2.1 is made by observing that $y_{1}=\xi(y)$ and $y_{2}=\xi f_{R}(y)$ are two possible outcomes of the reduction scheme $h_{\tau_{n}}$ applied to $y$. Although we cannot calculate $\bar{\delta}_{2}(y)=h_{0}(z)$ if we do not yet know what the obstructions of degree $n+1$ are, we do know that $f(z)=0$ because $f \zeta=f f_{L}=f f_{R}=f \theta=f \xi=f$. This says that " $z=0$ " expresses a linear relation valid in $G$ among the monomials in $M_{n}^{\prime}=\left\{x \in M_{n} \mid e(x)=n+1\right\}$, just as we knew in the algorithm that $y_{1}-y_{2}$ should be viewed as a new relation. We have given an explicit formula for what we mean by resolving the ambiguity presented by a 2 -chain, and we see that the formula comes directly from the differential $\bar{\delta}_{2}$ of $(8)$.

In practice one can continue as follows. Applying the function $\xi-\xi f_{R}$ to each degree $n+12$-chain in $V_{n}^{(2)}$, one obtains a set $D_{1}$ of relations, valid in $G$, among $M_{n}^{\prime}$. Applying $\xi$ to any of the original relations $\left\{\rho_{i}\right\}$ which have degree $n+1$ yields another set $D_{2}$ of relations in $M_{n}^{\prime} k$. When the subset $D_{1} \cup D_{2}$ of $M_{n}^{\prime} k$ is row reduced to standard row echelon form, the set $V \cap M_{n}^{\prime}$ of degree $n+1$ obstructions together with their straightening laws may be read off at once.

A final point of interest lies in an outline for an alternate proof of the diamond lemma for graded rings. How can we be sure that all of the obstructions are uncovered by the algorithm of Theorem 2.1? The alternate proof begins with the observations that $v-f(v)$ belongs to the ideal generated by $\rho=\left\{\rho_{i}\right\}$ for any $v \in V$ and that a subset of $\rho$, namely the minimal relations, can be identified with a basis for $\operatorname{Tor}_{2}^{G}(k, k) \approx\left(\operatorname{ker} \bar{\delta}_{1}\right) /\left(\operatorname{im} \bar{\delta}_{2}\right)$. If we include also one relation from $\rho$ for each nonminimal generator in $S$, a subset of $\rho$ serves as a basis for $\operatorname{im}\left(\bar{\delta}_{1}\right) \oplus \operatorname{Tor}_{2}^{G}(k, k) \approx$ 
$V k /\left(\operatorname{im} \bar{\delta}_{2}\right) . V k$ is spanned by a subset of $\rho$ and $\operatorname{im}\left(\bar{\delta}_{2}\right)$. Any obstruction can be obtained either from $\rho$ or from $\rho$ and $\operatorname{im}\left(\bar{\delta}_{2}\right)$. Since we have seen that $\bar{\delta}_{2}$ coincides with the process of resolving ambiguities, this is the desired conclusion.

3. Applications. The free resolution (1) is naturally suited for determining the homology of $k$-algebras. This section is devoted to several disparate results along those lines. After noting that a finite obstruction set renders each homology group finite over $k$ and generalizing a condition which assures $\operatorname{Tor}_{i}^{G}(k, k)=0$ for $i \geq 3$, we specialize to graded algebras. We obtain a bound on the ranks of the doubly graded homology modules for $G$ in terms of those for an associated monomial algebra. We outline a method for simplifying Hilbert series calculations. Lastly, we present a simpler than usual chain complex for computing the homology of the Steenrod algebra.

By the homology of an augmented $k$-algebra $G$ we mean the $k$-modules $\left\{\operatorname{Tor}_{i}^{G}(k, k)\right\}_{i \geq 0}$, where the augmentation gives both the right- and left-actions of $G$ on $k$. When each homology group of $G$ is a finite-dimensional vector space over $k$, the Poincaré series of $G$ is the formal power series

$$
P_{G}(y)=\sum_{i=0}^{\infty} \operatorname{Dim}_{k}\left(\operatorname{Tor}_{i}^{G}(k, k)\right) y^{i}=1+\cdots .
$$

There is no guarantee in general that the Poincaré series exists, i.e., that all $\operatorname{Tor}_{i}^{G}(k, k)$ are finite over $k$. When it does exist, various properties such as its growth rate, rationality or irrationality as an analytic function, and connection to other series can be studied to yield information about $G$. A series $\sum_{n=0}^{\infty} a_{n} z^{n}$ will be said to have exponential or slower growth if and only if there are constants $c_{1}, c_{2}>0$ such that $c_{2}\left(c_{1}\right)^{n} \geq a_{n} \geq 0$ for all $n$.

When $S$ is a locally well-ordered set of generators for $G$ and the set $V$ of obstructions is finite, the set of $n$-chains on $V$ is obviously finite for each $n$. Since these sets form a $k$-module basis for the chain complex obtained when the resolution (1) is tensored over $G$ with $k$, we have at once

LEMMA 3.1. If the obstruction set $V$ for the $k$-algebra $G$ relative to the locally well-ordered generating set $\left(S, e,<_{0}\right)$ is finite, then $\operatorname{Tor}_{i}^{G}(k, k)$ is finite-dimensional over $k$ for all $i \geq 2$. If in addition $S$ is finite, then $G$ has a Poincaré series with exponential or slower growth.

An interesting special case of Lemma 3.1 occurs when $G$ is finite-dimensional over $k$. Using other methods, Govorov fully analyzed this case in [9].

When $V$ is finite, the algorithm of $\S 2$ for successively approximating the obstruction set must stop yielding new obstructions after finitely many stages, and conversely if this happens at stage $m$ when

$$
m \geq \sup \left(\left\{e\left(r_{i}\right) \mid i \geq 1\right\} \cup\left\{2 e(v)-1 \mid v \in V_{m}\right\}\right),
$$

then all obstructions have been found. A delightful example is a variation on one suggested by Warren Dicks. Let $G=k\langle a, b\rangle /\langle a b a b a-a b a\rangle$, where the notation signifies that the quotient ideal is generated by the single element $(a b)^{2} a-a b a$. Automatically $a b a b a$ or a subword belongs to $V$. The two distinct ways of reducing the overlap ambiguity $a b a b a b a$ yield $h_{\tau}((a b a b a) b a)=h_{\tau}(a b a b a)=a b a$ and 
$h_{\tau}(a b(a b a b a))=h_{\tau}(a b a b a)=a b a$, so there are no further obstructions or ambiguities, i.e., $V=\{a b a b a\} . V^{(n)}$ is the singleton $\left\{a(b a)^{[(3 n+1) / 2]}\right\}$, brackets signifying the greatest integer function, so each $\operatorname{Tor}_{i}^{G}(k, k)$ for $i \geq 2$ has dimension zero or one. The actual Poincaré series, however, depends upon our choice of augmentation, and we give here only the answers. When $\varepsilon(a)=\varepsilon(b)=0, P_{G}(y)=y+(1-y)^{-1}$. When $\varepsilon(a) \neq 0$, but $\varepsilon(b)=0, P_{G}(y)=-y^{2}+(1-y)^{-1}$; and when $\varepsilon(a b)=1$, $P_{G}(y)=1+y$.

Another way to restrict the size of $V^{(n)}$ is by imposing combinatorial conditions on $V$. For $\left(S, e,<_{0}\right)$ locally well-ordered and $x \in k\langle S\rangle-(0)$, write $x=\sum_{i=1}^{m} c_{i} b_{i}$, $c_{i} \neq 0, b_{i} \in B$ distinct. The monomial $b_{j}$ is the high term of $x$ if and only if $b_{j} \geq b_{i}$ for $1 \leq i \leq m$. In $[\mathbf{1}]$, a set $\left\{a_{i}\right\}$ of monomials is defined to be combinatorially free if and only if $a_{i} \nsubseteq a_{j}$ for $i \neq j$ and $u a_{i}=a_{j} v$ cannot happen with $u, v \in B$ and $|u|<\left|a_{j}\right|$. The following theorem generalizes Theorem 3.2 of $[\mathbf{1}]$ in view of $[\mathbf{1}$, Corollary 2.12].

THEOREM 3.2. Suppose $G=k\langle S\rangle /\left\langle\alpha_{i}\right\rangle$, where $\left(S, e,<_{0}\right)$ is locally well-ordered and $\left\{\alpha_{i}\right\}_{i \in I}$ is any indexed subset of $k\langle S\rangle-(0)$. Letting $a_{i}$ be the high term of $\alpha_{i}$, suppose the set $A=\left\{a_{i}\right\}_{i \in I}$ is combinatorially free. Then $V=A$ and if $\varepsilon: G \rightarrow k$ is any augmentation, $\operatorname{Tor}_{i}^{G}(k, k)=0$ for $i \geq 3$.

PROOF. The combinatorially free condition is precisely equivalent to the nonexistence of inclusion or overlap ambiguities among the $\left\{a_{i}\right\}$. By [6] we have $V=A$. Since any 2-chain would violate the condition $u a_{i} \neq a_{j} v, V^{(2)}$ and consequently $V^{(n)}$ are empty. The conclusion follows from Theorem 1.4.

We henceforth suppose $G$ to be a connected graded $k$-algebra. There are graded free $G$-resolutions of $k$ (as in Theorem 1.5), so each of the homology groups $\operatorname{Tor}_{i}^{G}(k, k)$ is itself graded. Denoting by $\operatorname{Tor}_{p, q}^{G}(k, k)$ the degree $q$ component of $\operatorname{Tor}_{p}^{G}(k, k)$, we define the (double) Poincaré series of $G$ to be

$$
P_{G}(y, z)=\sum_{p, q \geq 0} \operatorname{Dim}_{k}\left(\operatorname{Tor}_{p, q}^{G}(k, k)\right) y^{p} z^{q} .
$$

When $G$ is generated by a locally finite graded set $(S, e)$, each $V^{(n)} k$ is a locally finite graded $k$-module, so the double Poincaré series automatically exists; this has traditionally been deduced also from the bar resolution.

The simplest graded algebras from a combinatorial standpoint are those whose ideal of relations is generated by monomials on a set of generators $S$. If $\rho \subseteq B$ is any subset, then clearly $x \in B-M$ if and only if $v \subseteq x$ for some $v \in \rho$. Thus $V$ is precisely the antichain of all minimal (in the sense of $\subseteq$ ) monomials in $\rho$. In particular, $\#(V) \leq \#(\rho)$. Monomial algebras, as algebras with monomial relations are sometimes called, are distinguished by the property that $f: k\langle S\rangle \rightarrow G \approx M k$ has $f(x)=x$ for $x \in M$ and $f(x)=0$ for $x \in B-M$. From this we deduce

LEMMA 3.3. Let $G=k\langle S\rangle /\langle\rho\rangle$ be a monomial algebra, where $\rho \subseteq B$ is a set of monomials on $S$, and let $\varepsilon(x)=0$ for all $x \in S$. Suppose $S$ contains no superfluous generators, i.e., $S \cap \rho$ is empty. Then $V=\{v \in \rho \mid$ no $u \in \rho$ is a proper submonomial of $v\}$ and in the resolution (1),

$$
\delta_{n}\left(x_{i_{1}} \cdots x_{i_{b_{n}}}\right)=x_{i_{1}} \cdots x_{i_{b_{n-1}}} \otimes x_{i_{b_{n-1}+1}} \cdots x_{i_{b_{n}}} .
$$

In particular, $\operatorname{Tor}_{i}^{G}(k, k) \approx V^{(i-1)} k$ as graded $k$-modules for all $i \geq 0$. 
PROOF. Tracing the construction in Theorem 1.4 under these circumstances, we find inductively that (11) holds and that the splitting $i_{n}: \operatorname{ker}\left(\delta_{n-1}\right) \rightarrow V^{(n)} k \otimes G$ is given by

$$
i_{n}\left(x_{i_{1}} \cdots x_{i_{b_{n-1}}} \otimes x_{i_{b_{n-1}+1}} \cdots x_{i_{b_{n}}} \cdots x_{i_{s}}\right)=x_{i_{1}} \cdots x_{i_{b_{n}}} \otimes x_{i_{b_{n}+1}} \cdots x_{i_{s}}
$$

when $x_{i_{b_{n-1}+1}} \cdots x_{i_{s}}$ and consequently $x_{i_{b_{n}+1}} \cdots x_{i_{s}}$ belong to $M$. In view of (11), all differentials reduce to zero after tensoring over $G$ with $k$.

Monomial rings were considered by Govorov [8] and Backelin [3]. Backelin computed the Poincaré series of monomial algebras by constructing a free left $G$ resolution of $k$ which is equivalent in every way to our right resolution (1) with differential (11). He used this resolution to show that the double (in fact, $(\#(S)+1)$ variable!) Poincaré series of a finitely generated monomial ring represents a rational function if the set of relations is finite.

Even when $G$ is not a monomial ring, we can use the resolution (1) to obtain bounds on $\operatorname{Tor}_{p, q}^{G}(k, k)$. As usual, let $V$ be the set of obstructions for $G$ and $M$ the o.i.m. which serves as a basis. $V$ being a set of monomials, we define the ring $k\langle S\rangle /\langle V\rangle$ to be the associated monomial ring to $\left(G, S, e,<_{0}\right)$. We call the graded $k$-algebra $A=A(G)$ "an associated monomial ring" to $G$ if it equals $k\langle S\rangle /\langle V\rangle$ for some choice of locally well-ordered generating set $\left(S, e,<_{0}\right)$ for $G$.

LEMMA 3.4. Let $G$ be a connected graded $k$-algebra with a locally finite set of generators $\left(S, e,<_{0}\right)$. Let $A(G)$ be the associated monomial ring to $\left(G, S, e,<_{0}\right)$. Then $P_{G}(y, z) \leq P_{A(G)}(y, z)$, inequality holding coefficientwise.

Proof. This is immediate from Theorem 1.5 because by Lemma 3.3 , the degree $q$ component of $V^{(p-1)} k$ is $\operatorname{Tor}_{p, q}^{A(G)}(k, k)$.

We shall return in $\S 4$ to this idea of using a monomial ring's Poincaré series to bound that of an arbitrary graded ring.

When $A(G)$ is an associated monomial ring to $G$, the same o.i.m. $M$ serves as a basis for $G$ and for $A(G)$, so in each degree their dimensions over $k$ coincide. Once again we may summarize this information in a series: writing a graded $k$-module $N$ as $N=N_{0} \oplus N_{1} \oplus N_{2} \oplus \cdots$, the Hilbert series of $N$ is

$$
H_{N}(z)=\sum_{n=0}^{\infty} \operatorname{Dim}_{k}\left(N_{n}\right) z^{n}
$$

defined if each $N_{n}$ is finite over $k$. For $G$ a connected graded algebra, $H_{G}(z)$ exists if and only if $G$ has a locally finite set of generators. In view of [1, Lemma 1.2] we always have coefficientwise inequality

$$
H_{G}(z) \leq\left(1-\sum_{x \in S} z^{|x|}\right)^{-1}
$$

so Hilbert series of finitely generated graded algebras have exponential or slower growth. Rephrased, our previous observation about associated monomial rings may be written

$$
H_{G}(z)=H_{A(G)}(z)
$$

when either side is defined. 
When $H_{G}$ exists, a well-known connection between Hilbert and Poincaré series is deduced from a minimal $G$-resolution of $k$,

$$
0 \leftarrow k \leftarrow G \leftarrow U_{1} \otimes G \leftarrow U_{2} \otimes G \leftarrow U_{3} \otimes G \leftarrow \cdots,
$$

in which $U_{i} \approx \operatorname{Tor}_{i}^{G}(k, k)$ as graded $k$-modules. Because $G$ is locally finite, the alternating sum

$$
\sum_{i=0}^{\infty}(-1)^{i} H_{U_{i}}(z) \cdot H_{G}(z)
$$

is defined, and because (13) is exact it equals $H_{k}(z)=1$. The sum (14) is quickly rewritten as

$$
H_{G}(z) \cdot\left[\sum_{i=0}^{\infty}(-1)^{i} \sum_{j=0}^{\infty} \operatorname{Dim}_{k}\left(\operatorname{Tor}_{i, j}^{G}(k, k)\right) z^{j}\right]
$$

yielding

$$
H_{G}(z) \cdot P_{G}(-1, z)=1 \text {. }
$$

In view of (12) and (15) and Lemma 3.3 applied to $A(G)$, we may write

$$
H_{G}(z)=\left[1-\sum_{n=0}^{\infty}(-1)^{n} H_{V^{(n)} k}(z)\right]^{-1},
$$

where $V$ is the obstruction set for $G$ relative to $\left(S, e,<_{0}\right)$. This offers a surprisingly efficient algorithm for computation of Hilbert series. Given a connected graded algebra $G$ via a locally finite generating set $\left(S, e,<_{0}\right)$ and homogeneous relation set $\rho$, first determine the set of obstructions of degree $\leq m$ by the methods of $\S 2$. Then form all $n$-chains on this set for $n \leq m$, count the number in each degree up to $m$, and take an alternating sum of the resulting power series. $H_{G}(z)$ agrees with the formal inverse of the result in degrees not exceeding $m$. Although it appears elementary, this can be a very good way of calculating or checking a Hilbert series, especially for algebras with few relations. For example, it simplifies the total amount of work needed to verify the transcendental Hilbert series of the "Shearer-type" algebras listed in [2, Theorem 5].

Lastly, we consider situations in which, for various reasons, the obstruction set behaves particularly well. Suppose $\left(S, e,<_{0}\right)$ is locally finite and the obstruction set $V$ consists entirely of quadratic monomials. Then any $n$-chain has length $n+1$. If the length grading on $S$ is compatible with the grading of $G$, then $V^{(n)} k$ is concentrated in degree $(n+1)$, and $\delta_{n}$ must reduce to zero after tensoring over $G$ with $k$. Consequently $\operatorname{Tor}_{p, q}^{G}(k, k)=0$ for $q \neq p$; this is one of the equivalent conditions for $G$ to be a "Fröberg" or "Koszul" algebra [5, 12], about which many results are known. The condition we have given here is also the right-module version of the PBW condition described in [12], so we shall not elaborate on it further.

Let us turn our attention to $A_{p}$, the Steenrod algebra mod $p$. One of the special properties of $A_{p}$ is that it has a basis consisting of admissible sequences, and in our terminology the admissible sequences form an o.i.m. Therefore the obstruction set is precisely known. 
The Adem relations provide us with the obstruction set and straightening rules:

$$
\begin{gathered}
(p=2) \quad \mathrm{Sq}^{a} \mathrm{Sq}^{b}=\sum_{t=0}^{[a / 2]}\left(\begin{array}{c}
b-t-1 \\
a-2 t
\end{array}\right) \mathrm{Sq}^{a+b-t} \mathrm{Sq}^{t} \quad \text { if } a<2 b . \\
\beta^{2}=0, \\
(p>2) \\
P^{a} P^{b}=\sum_{t=0}^{[a / p]}(-1)^{a+t}\left(\begin{array}{c}
(p-1)(b-t)-1 \\
a-p t
\end{array}\right) P^{a+b-t} P^{t} \quad \text { if } a<p b, \\
P^{a} \beta P^{b}=\sum_{t=0}^{[a / p]}(-1)^{a+t}\left(\begin{array}{c}
(p-1)(b-t)-1 \\
a-p t
\end{array}\right) \beta P^{a+b-t} P^{t} \\
\quad+\sum_{t=0}^{[(a-1) / p]}(-1)^{a+t}\left(\begin{array}{c}
(p-1)(b-t)-1 \\
a-p t-1
\end{array}\right) P^{a+b-t} \beta P^{t} \quad \text { if } a \leq p b .
\end{gathered}
$$

In order for these to be proper straightening rules in the sense of $\S 1$, we must order the set of generators such that the left-hand monomial of each equation exceeds the terms on the right. When $p=2$, this necessitates taking $\mathrm{Sq}^{1}>_{0} \mathrm{Sq}^{2}>_{0} \mathrm{Sq}^{3}>_{0} \cdots$ and when $p>2, P^{1}>_{0} P^{2}>_{0} P^{3}>_{0} \cdots>_{0} \beta$. This is a good example of a situation in which it pays to have $\left(S, e,<_{0}\right)$ be locally well-ordered but not wellordered.

We could proceed at once to apply Theorem 1.4, but we note first that half the generators when $p=2$ can conveniently be omitted. Using $\mathrm{Sq}^{2 m+1}=\mathrm{Sq}^{1} \mathrm{Sq}^{2 m}$, (17) becomes

$$
\begin{aligned}
\mathrm{Sq}^{2 m} \mathrm{Sq}^{b}= & \sum_{t=0}^{m}\left(\begin{array}{c}
b-t-1 \\
2 m-2 t
\end{array}\right) \mathrm{Sq}^{2 m+b-t} \mathrm{Sq}^{t} \\
= & \mathrm{Sq}^{1} \sum_{\substack{0 \leq t \leq m \\
b-t \text { odd }}}\left(\begin{array}{c}
b-t-1 \\
2 m-2 t
\end{array}\right) \mathrm{Sq}^{2 m+b-t-1} \mathrm{Sq}^{t} \\
& +\sum_{\substack{0 \leq t \leq m \\
b-t \text { even }}}\left(\begin{array}{c}
b-t-1 \\
2 m-2 t
\end{array}\right) \mathrm{Sq}^{2 m+b-t} \mathrm{Sq}^{t}, \\
\mathrm{Sq}^{1} \mathrm{Sq}^{2 m} \mathrm{Sq}^{b}= & \mathrm{Sq}^{2 m+1} \mathrm{Sq}^{b}=\sum_{t=0}^{m}\left(\begin{array}{c}
b-t-1 \\
2 m-2 t+1
\end{array}\right) \mathrm{Sq}^{2 m+1+b-t} \mathrm{Sq}^{t} \\
= & \mathrm{Sq}^{1} \sum_{\substack{0 \leq t \leq m \\
b-t \text { even }}}\left(\begin{array}{c}
b-t-1 \\
2 m-2 t+1
\end{array}\right) \mathrm{Sq}^{2 m+b-t} \mathrm{Sq}^{t},
\end{aligned}
$$

the simplification in (19b) arising because a binomial coefficient representing even choose odd is always even. When $b-t$ is even, moreover, $\left(\begin{array}{c}b-t-1 \\ 2 m-2 t\end{array}\right)$ and $\left(\begin{array}{c}b-t-1 \\ 2 m-2 t+1\end{array}\right)$ have the same parity, which implies that $(19 \mathrm{~b})$ is obtained by multiplying $(19 \mathrm{a})$ on 
the left by $\mathrm{Sq}^{1}$ and using $\left(\mathrm{Sq}^{1}\right)^{2}=0$. Thus $(19 \mathrm{~b})$ is a superfluous relation and (17) may be replaced by

$$
\begin{gathered}
\left(\mathrm{Sq}^{1}\right)^{2}=0 \\
\mathrm{Sq}^{2 a} \mathrm{Sq}^{2 b}=\mathrm{Sq}^{1} \sum_{\substack{0 \leq t \leq a \\
t \text { odd }}}\left(\begin{array}{c}
2 b-t-1 \\
2 a-2 t
\end{array}\right) \mathrm{Sq}^{2 a+2 b-t-1} \mathrm{Sq}^{1} \mathrm{Sq}^{t-1} \\
+\sum_{\substack{0 \leq t \leq a \\
t \text { even }}}\left(\begin{array}{c}
2 b-t-1 \\
2 a-2 t
\end{array}\right) \mathrm{Sq}^{2 a+2 b-t} \mathrm{Sq}^{t} \quad \text { if } a<2 b \\
\mathrm{Sq}^{2 a} \mathrm{Sq}^{1} \mathrm{Sq}^{2 b}= \\
\sum_{\substack{0 \leq t \leq a \\
t \text { odd }}}\left(\begin{array}{c}
2 b-t \\
2 a-2 t
\end{array}\right) \mathrm{Sq}^{2 a+2 b+1-t} \mathrm{Sq}^{1} \mathrm{Sq}^{t-1} \\
+\operatorname{Sq}^{1} \sum_{\substack{0 \leq t \leq a \\
t \text { even }}}\left(\begin{array}{c}
2 b-t \\
2 a-2 t
\end{array}\right) \mathrm{Sq}^{2 a+2 b-t} \mathrm{Sq}^{t} \quad \text { if } a \leq 2 b .
\end{gathered}
$$

In order to use (20) as our straightening rules, take $S=\left\{\mathrm{Sq}^{1}\right\} \cup\left\{\mathrm{Sq}^{2 a} \mid a \geq 1\right\}$, ordered by $\mathrm{Sq}^{2}>_{0} \mathrm{Sq}^{4}>_{0} \mathrm{Sq}^{6}>_{0} \cdots>_{0} \mathrm{Sq}^{1}$.

In view of the similarity between (18) and (20), we let $\beta$ denote $\mathrm{Sq}^{1}$ and $P^{i}$ denote $\mathrm{Sq}^{2 i}$ when $p=2$. Whether $p$ is even or odd, we may write $S=\{\beta\} \cup\left\{P^{i} \mid i \geq 1\right\}$ with $e(\beta)=1$ and $e\left(P^{i}\right)=2(p-1) i$. Whether $p$ is even or odd,

$$
V=\left\{\beta^{2}\right\} \cup\left\{P^{i} P^{j} \mid i<p j\right\} \cup\left\{P^{i} \beta P^{j} \mid i \leq p j\right\}
$$

and consequently

$$
V^{(n-1)}=\left\{\beta^{n}\right\} \cup\left\{P^{i_{1}} \beta^{\sigma_{1}} P^{i_{2}} \beta^{\sigma_{2}} \cdots \beta^{\sigma_{n-1}} P^{i_{n}} \mid \sigma_{j}=0 \text { or } 1, i_{j}<\sigma_{j}+p i_{j+1}\right\} .
$$

Theorem 1.4 yields at once

THEOREM 3.5. Let $p$ be any prime and set $k=\mathbf{Z} / p \mathbf{Z}$. Let $U_{0}=k, U_{1}=S k$, and for $n \geq 2 U_{n}=V^{(n-1)} k$, with $S$ and $V^{(n-1)}$ as above. Then there exists $a$ chain complex

$$
\cdots \stackrel{\partial_{n+1}}{\rightarrow} U_{n} \stackrel{\partial_{n}}{\rightarrow} U_{n-1} \stackrel{\partial_{n-1}}{\rightarrow} \cdots \stackrel{\partial_{1}}{\rightarrow} U_{0} \rightarrow 0
$$

of graded $k$-modules such that

$$
\left(H_{i}\left(U_{*}, \partial_{*}\right)\right)_{j} \approx \operatorname{Tor}_{i, j}^{A_{p}}(k, k) .
$$

Our claim that (21) is smaller than the usual $\Lambda$-algebra is based on the fact that we have gotten by with a smaller set of generators, e.g., Priddy [12] used $S=\left\{P^{i} \mid i \geq 1\right\} \cup\left\{\beta P^{i} \mid i \geq 0\right\} . V^{(n-1)}$ is smaller than its counterpart in the usual basis in that the "spikes" $\beta^{s} P^{i_{1}} \beta^{\sigma_{1}} \ldots P^{i_{n}}$, where $s>0$, have been eliminated. Hopefully Theorem 3.5 will be of use for actual homology calculations. A word of warning: in spite of the apparent similarity between the $p=2$ and $p$ odd cases, formulas (18) and (20), which determine the differentials $\left\{\partial_{n}\right\}$, are very different.

Finally, the multiplication in $\operatorname{Ext}_{\mathcal{A}_{p}}^{*, *}(k, k)$ (or comultiplication in $\operatorname{Tor}_{*, *}^{\mathcal{A}_{p}}(k, k)$ ) can be obtained from (21) using Sjödin's analysis of the way in which the Yoneda product is calculated from an arbitrary projective resolution $[\mathbf{1 3}]$. 
4. A bound on the homology of commutative graded rings. We turn our attention now to commutative graded algebras. We shall prove a precise analog of Lemma 3.4. Combining this with a result due to Backelin, we obtain a bound on the range of internal degrees in which an algebra's homology may be nonzero.

Let $R$ be a finitely generated commutative connected graded $k$-algebra. Because $R$ is Noetherian, each $\operatorname{Tor}_{p}^{R}(k, k)$ is finite-dimensional over $k$, and this means that for each $p \geq 0$, $\operatorname{Tor}_{p, q}^{R}(k, k)$ can be nonzero for only finitely many $q$. The connectedness of $R$ assures that $\operatorname{Tor}_{p, q}^{R}(k, k)=0$ for $p<q$, but in general infinitely many $\operatorname{Tor}_{p, p}^{R}(k, k)$ can be nonzero. Unless $R$ is regular, $\operatorname{Tor}_{p}^{R}(k, k) \neq 0$ for all $p$, hence

$$
q_{0}(p, R)=\sup \left\{q \mid \operatorname{Tor}_{p, q}^{R}(k, k) \neq 0\right\}
$$

is well-defined. We seek a bound on $q_{0}(p, R)$.

The number $q_{0}(1, R)$ is the highest degree of any generator which belongs to a minimal set of generators for $R$, and likewise $q_{0}(2, R)$ is the maximal degree of a minimal relation. $q_{0}(3, R)$ can be interpreted as a bound on the complexity of the syzygies among the relations of $R$, and some estimates exist for it (e.g., see [10]). The behavior of $q_{0}(p, R)$ for $p \geq 4$ has up to now remained largely unknown. Our goal here is to prove for every nonregular $R$ that $q_{0}(p, R)$ is at most linear in $p$.

We proceed in a manner analogous to that of Lemma 1.1. Although in this paper we postponed commutative rings until last, historically Macaulay [11] analyzed the commutative situation long before the noncommutative situation was even considered. The ideas we recall next inspired our earlier results rather than vice versa.

Let $\left(S, e,<_{0}\right)$ be a finite totally ordered graded set of homogeneous generators for $R$ as a $k$-algebra. Let $\mathbf{N}$ denote the nonnegative integers, write $S=\left\{x_{1}, \ldots, x_{m}\right\}$ with $x_{i}<_{0} x_{j}$ if $i<j$, and let $C=\mathbf{N}^{m}$. Let $g: k\left[x_{1}, \ldots, x_{m}\right] \rightarrow R$ be the surjection and write $x^{\alpha}$ for $x_{1}^{\alpha_{1}} \cdots x_{m}^{\alpha_{m}}$ in $k\left[x_{1}, \ldots, x_{m}\right]$ when $\alpha=\left(\alpha_{1}, \ldots, \alpha_{m}\right) \in C$. $C$ is a monoid under addition and $e: C \rightarrow \mathbf{N}$ is given by $e(\alpha)=\alpha_{1} e\left(x_{1}\right)+\cdots+\alpha_{m} e\left(x_{m}\right)$ if $\alpha=\left(\alpha_{1}, \ldots, \alpha_{m}\right)$. We adopt Macaulay's reverse lexicographic ordering $C$ : for $\alpha=\left(\alpha_{1}, \ldots, \alpha_{m}\right)$ and $\beta=\left(\beta_{1}, \ldots, \beta_{m}\right)$, set $\alpha<\beta$ if $|\alpha|<|\beta|$. When $|\alpha|=|\beta|$, set $\alpha<\beta$ if and only if $\alpha_{j}>\beta_{j}$ for some $j$ while $\alpha_{i}=\beta_{i}$ for $i<j$. This ordering makes $(C,<)$ isomorphic as an ordered set with the natural numbers.

Write $\alpha \Delta \beta$ if and only if $x^{\alpha}$ divides $x^{\beta}$ in $k\left[x_{1}, \ldots, x_{m}\right]$. The relation $\Delta$ is a partial ordering on $C$ and $\alpha \triangle \beta$ if and only if $\beta=\alpha+\gamma$ for some $\gamma \in C$. Note that for any $\gamma, \alpha<\beta$ implies $\alpha+\gamma<\beta+\gamma$ and $\alpha \Delta \beta$ implies $(\alpha+\gamma) \triangle(\beta+\gamma)$.

Macaulay set $L=\left\{\alpha \in C \mid g\left(x^{\alpha}\right) \notin \operatorname{Span}\left(g\left(x^{\beta}\right) \mid \beta<\alpha\right)\right\}$ and observed that $\left\{g\left(x^{\alpha}\right) \mid \alpha \in L\right\}$ is a $k$-basis for $R$. Furthermore, $\alpha \in L$ and $\beta \triangle \alpha$ imply $\beta \in L$. Define the (commutative) associated monomial ring to $R$ relative to $\left(S, e,<_{0}\right)$ to be the quotient ring $A=k\left[x_{1}, \ldots, x_{m}\right] /\left[x^{\beta} \mid \beta \notin L\right]$. Because the images of $x^{\alpha}, \alpha \in L$, are a $k$-basis for $A, H_{A}(z)=H_{R}(z)$, an analog of (12). We prove next an analog of Lemma 3.4.

LEMMA 4.1. Let $\left(S, e,<_{0}\right)$ be a finite ordered graded set of generators for the commutative connected graded $k$-algebra $R$, and let $A$ be the associated monomial ring. There is a spectral sequence starting with $\operatorname{Tor}_{*, *}^{A}(k, k)$ and converging to $\operatorname{Tor}_{*, *}^{R}(k, k)$. In particular,

$$
\operatorname{Dim}_{k}\left(\operatorname{Tor}_{p, q}^{R}(k, k)\right) \leq \operatorname{Dim}_{k}\left(\operatorname{Tor}_{p, q}^{A}(k, k)\right)
$$


for each $p$ and $q$. Equivalently, we have coefficientwise inequality in $P_{R}(y, z) \leq$ $P_{A}(y, z)$.

PROOF. Let $R_{+}=R_{1} \oplus R_{2} \oplus R_{3} \oplus \cdots=\operatorname{ker}(\varepsilon: R \rightarrow k)$ and let

$$
0 \stackrel{d_{0}=0}{\leftarrow} k \stackrel{d_{1}=0}{\longleftarrow} R_{+} \stackrel{d_{2}}{\leftarrow} R_{+} \otimes R_{+} \stackrel{d_{3}}{\longleftarrow} R_{+} \otimes R_{+} \otimes R_{+} \stackrel{d_{4}}{\leftarrow} \ldots
$$

with

$$
d_{n}\left(u_{1} \otimes \cdots \otimes u_{n}\right)=\sum_{j=1}^{n-1}(-1)^{j-1} u_{1} \otimes \cdots \otimes u_{j} u_{j+1} \otimes \cdots \otimes u_{n}
$$

be the chain complex obtained from the bar resolution, where

$$
\operatorname{Tor}_{p, *}^{R}(k, k) \approx \operatorname{ker}\left(d_{p}\right) / \operatorname{im}\left(d_{p+1}\right) .
$$

Taking $C, g, L, A$, as above and $L_{+}=L-\{(0, \ldots, 0)\}$, let $w_{\alpha}=g\left(x^{\alpha}\right)$ for $\alpha \in L$, so $\left\{w_{\alpha_{(1)}} \otimes \cdots \otimes w_{\alpha_{(n)}} \mid \alpha_{(i)} \in L_{+}\right\}$is a basis for $\left(R_{+}\right)^{\otimes n}$. Set $W_{n}=\left(R_{+}\right)^{\otimes n}$ and for $\alpha \in C$,

$$
W_{n, \alpha}=\operatorname{Span}\left\{w_{\alpha_{(1)}} \otimes \cdots \otimes w_{\alpha_{(n)}} \mid \alpha_{(i)} \in L_{+}, \alpha_{(1)}+\cdots+\alpha_{(n)}=\alpha\right\} .
$$

Note that $W_{n}=\bigoplus_{\alpha \in C} W_{n, \alpha}$ for each $n$.

For $\alpha, \beta \in L$, consider the product $w_{\alpha} w_{\beta}$ in $R$. If $\alpha+\beta \in L$, then $w_{\alpha} w_{\beta}=$ $g\left(x^{\alpha}\right) g\left(x^{\beta}\right)=g\left(x^{\alpha+\beta}\right)=w_{\alpha+\beta}$. If $\alpha+\beta \notin L$, then

$$
\begin{aligned}
w_{\alpha} w_{\beta} & =g\left(x^{\alpha+\beta}\right) \in \operatorname{Span}\left\{g\left(x^{\gamma}\right) \mid \gamma<\alpha+\beta\right\} \\
& =\operatorname{Span}\left\{w_{\gamma} \mid \gamma \in L, \gamma<\alpha+\beta\right\} .
\end{aligned}
$$

Furthermore, $\alpha+\beta \notin L$ if and only if $x^{\alpha+\beta}$ is zero in the commutative associated monomial ring $A$. Summarizing, we have

$$
\left\{\begin{array}{l}
w_{\alpha} w_{\beta}=w_{\alpha+\beta} \text { if } x^{\alpha} x^{\beta}=x^{\alpha+\beta} \text { in } A \\
w_{\alpha} w_{\beta} \in \operatorname{Span}\left\{w_{\gamma} \mid \gamma \in L, \gamma<\alpha+\beta\right\}
\end{array} \text { if } x^{\alpha} x^{\beta}=0 \text { in } A .\right.
$$

The key observation for the proof is that (22) is filtered as a chain complex by $C$. Let $F_{n, \beta}=\bigoplus_{\alpha \leq \beta} W_{n, \alpha}$. If $w=w_{\alpha_{(1)}} \otimes \cdots \otimes w_{\alpha_{(n)}} \in W_{n, \beta}$, then

$$
d_{n}(w)=\sum_{j=1}^{n-1}(-1)^{j-1} w_{\alpha_{(1)}} \otimes \cdots \otimes w_{\alpha_{(j)}} w_{\alpha_{(j+1)}} \otimes \cdots \otimes w_{\alpha_{(n)}},
$$

and in view of $(24)$, each summand belongs to $F_{n-1, \beta}$. Since $(C,<)$ is isomorphic to the natural numbers, the filtration $\left\{F_{*, *}\right\}$ gives rise to an ordinary spectral sequence of graded $k$-modules converging to $H_{*}\left(W_{*}, d_{*}\right)=\operatorname{Tor}_{*, *}^{R}(k, k)$.

To recognize the initial term of the spectral sequence, note that it equals $H_{*}\left(W_{*}, \bar{d}_{*}\right)$, where

$$
\bar{d}_{n}(w)=\sum_{j=1}^{n-1}(-1)^{j-1} w_{\alpha_{(1)}} \otimes \cdots \otimes \mu\left(w_{\alpha_{(j)}}, w_{\alpha_{(j+1)}}\right) \otimes \cdots \otimes w_{\alpha_{(n)}},
$$

and

$$
\mu\left(w_{\alpha}, w_{\beta}\right)= \begin{cases}w_{\alpha+\beta} & \text { if } w_{\alpha} w_{\beta}=w_{\alpha+\beta} \text { in } R \\ 0 & \text { if } w_{\alpha} w_{\beta} \in \operatorname{Span}\left\{w_{\gamma} \mid \gamma<\alpha+\beta\right\} \text { in } R .\end{cases}
$$

In view of $(24)$ and $(23),\left(W_{*}, \bar{d}_{*}\right)$ is isomorphic to the chain complex which is like (22) but is obtained from the bar resolution for $A$ instead of $R$. The initial term of the spectral sequence is precisely $\operatorname{Tor}_{*, *}^{A}(k, k)$. 
THEOREM 4.2. Let $R$ be a nonregular commutative Noetherian connected graded $k$-algebra. There exists a constant $c=c(R)$ such that $p \leq q_{0}(p, R) \leq c p$ for all $p \geq 0$.

ProOF. Letting $A$ be the commutative associated monomial ring relative to some finite generating set $\left(S, e,<_{0}\right)$, we have $q_{0}(p, R) \leq q_{0}(p, A)$ for each $p$ by Lemma 4.1, so it suffices to prove the result for commutative monomial rings. Backelin [4] has recently shown that any commutative Noetherian connected graded ring whose ideal of relations is generated by monomials has a rational double Poincaré series. In particular, it has the form

$$
P_{A}(y, z)=\frac{(1+y z)^{m}}{1+\sum_{p, q>0} a_{p q} y^{p} z^{q}}
$$

for some integers $\left\{a_{p q}\right\}$ almost all zero, where $m=\#(S)$. Let

$$
c(A)=\sup \left\{1 ; q / p \mid a_{p q} \neq 0\right\}<\infty .
$$

Formal expansion of the right-hand side of (25) shows that a nonzero term $b_{p q} y^{p} z^{q}$ in $P_{A}(y, z)-1$ must have $q / p \leq c=c(A)$ and consequently $q_{0}(p, A) \leq c p$, as desired.

\section{REFERENCES}

1. D. Anick, Non-commutative graded algebras and their Hilbert series, J. Algebra 78 (1982), 120-140.

2. D. Anick and C. Löfwall, Hilbert series of finitely presented algebras, Algebra, Algebraic Topology, and their Interactions, Lecture Notes in Math., vol. 1183, Springer-Verlag, pp. $32-55$.

3. J. Backelin, La série de Poincaré-Betti d'une algèbre graduée de type fini à une relation est rationnelle, C. R. Acad. Sci. Paris. Sér. A 287 (1978), 843-846.

4. __ Les anneaux locaux á relations monomiales ont des séries de Poincaré-Betti rationnelles, C. R. Acad. Sci. Paris Sér. I 295 (1982), 607-610.

5. J. Backelin and R. Fröberg, Koszul algebras, Veronese subrings, and rings with linear resolutions, Rev. Roumaine Math. Pures Appl. 30 (1985), 85-97.

6. G. Bergman, The diamond lemma for ring theory, Adv. in Math. 29 (1978), 178-218.

7. D. B. A. Epstein and N. E. Steenrod, Cohomology operations, Ann. of Math. Studies, No. 50, Princeton Univ. Press, Princeton, N.J., 1962.

8. V. E. Govorov, Graded algebras, Math. Notes 12 (1972), 552-556.

9. __ Dimension and multiplicity of graded algebras, Siberian Math. J. 14 (1973), 12001206.

10. D. Lazard, Algèbre linéaire sur $k\left[x_{1}, \ldots, x_{n}\right]$ et élimination, Bull. Soc. Math. France 105 (1977), 165-190.

11. F. S. Macaulay, Some properties of enumeration in the theory of modular systems, Proc. London Math. Soc. 26 (1927), 531-555.

12. S. Priddy, Koszul resolutions, Trans. Amer. Math. Soc. 152 (1970), 39-77.

13. G. Sjödin, $A$ set of generators for $\operatorname{Ext}_{R}(k, k)$, Math. Scand. 38 (1976), 199-210.

14. R. P. Stanley, Hilbert functions of graded algebras, Adv. in Math. 28 (1978), 57-83.

Department of MATHEMATICS, Massachusetts Institute of Technology, CAMBRIDGE, MASSACHUSETTS 02139 